\section{Spotting ligandable lysines}

A method to detect highly reactive lysine residues in the proteome helps spot those ripe for targeting by covalent ligands.

Lysine residues are commonly found on protein surfaces, where they help mediate protein-protein interactions, and at active sites of enzymes, where they play roles in catalytic function. The reactivity of lysine residues across whole proteomes remains largely a mystery, however.

Researchers at The Scripps Research Institute recently developed a chemical proteomics approach to capture a broad view of lysine functionality across the human proteome. The method may assist chemical biologists developing probes for studying the functions of proteins of interest and drug designers developing new molecules for treating disease.

The method, developed in Benjamin Cravatt's lab, relies on a pentynoic acid sulfotetrafluorophenyl ester probe that specifically reacts with amines found on accessible lysine residues in proteins. The researchers designed the probe to contain an alkyne moiety, which enabled them to attach biotin via a click-chemistry reaction. This allowed them to enrich reactive lysinecontaining proteins using streptavidin-based affinity chromatography, digest the proteins into peptides, and identify the peptides by liquid chromatography-mass spectrometry.

Cravatt's team assessed the reactivity of thousands of lysine residues in multiple human cancer cell lines. A small subset of lysines showed an especially strong affinity for their probe, and they dubbed these lysines 'hyper-reactive.' These lysines tended to be present at known functional sites in enzymes and in protein pockets. When the researchers mutated a hyper-reactive lysine to arginine in a pocket far from the active site of of platelet-type phosphofructokinase, its catalytic activity was reduced, which indi- cated that the lysine may play a role in allosteric regulation.

By allowing their amine-reactive probe to compete with a small library of aminereactive molecular fragments, the team screened for the covalent targeting potential, or 'ligandability', of lysines proteome wide. They identified 121 ligandable lysines in 113 proteins and followed up with detailed functional analysis of several ligand-lysine interactions. Some of these targets, including transcription factors, lack deep pockets and have been considered undruggable by small molecules. The work by the Cravatt team therefore outlines a promising way forward to help expand the druggable proteome.

\section{Allison Doerr}

\section{RESEARCH PAPERS}

Hacker, S.M. et al. Global profiling of lysine reactivity and ligandability in the human proteome. Nat. Chem. http://dx.doi.org/10.1038/nchem.2826 (2017). 